\title{
Infants' visual and auditory communication when a partner is or is not visually attending
}

\author{
Ulf Liszkowski*, Konstanze Albrecht, Malinda Carpenter, Michael Tomasello \\ Max Planck Institute for Evolutionary Anthropology, Department of Developmental and Comparative Psychology, \\ Deutscher Platz 6, 04103 Leipzig, Germany \\ Received 22 January 2007; received in revised form 6 July 2007; accepted 12 October 2007
}

\begin{abstract}
In the current study we investigated infants' communication in the visual and auditory modalities as a function of the recipient's visual attention. We elicited pointing at interesting events from thirty-two 12-month olds and thirty-two 18-month olds in two conditions: when the recipient either was or was not visually attending to them before and during the point. The main result was that infants initiated more pointing when the recipient's visual attention was on them than when it was not. In addition, when the recipient did not respond by sharing interest in the designated event, infants initiated more repairs (repeated pointing) than when she did, again, especially when the recipient was visually attending to them. Interestingly, accompanying vocalizations were used intentionally and increased in both experimental conditions when the recipient did not share attention and interest. However, there was little evidence that infants used their vocalizations to direct attention to their gestures when the recipient was not attending to them.
\end{abstract}

CC 2007 Elsevier Inc. All rights reserved.

Keywords: Pointing; Vocalizing; Infant communication; Understanding attention; Communicative looks; Message repair; Cross-modal communication

Infants begin pointing at interesting things and events around their first birthdays. With regard to the underlying cognitive and motivational abilities, there has been much debate over 'rich' and 'lean' interpretations (see e.g., Bates, 1979; Camaioni, Perucchini, Bellagamba, \& Colonnesi, 2004; Gómez, Sarria, \& Tamarit, 1994; Liszkowski, 2006; Moore \& Corkum, 1994; Tomasello, Carpenter, \& Liszkowski, 2007). But even more basically, there has also been debate over the extent to which there is good evidence that young infants point with the intent to communicate at all. For example, it has been suggested that infants point initially without communicative intent for the self, and that parents only respond to their pointing as if it was communicative (see e.g., Bates, 1979; Carpendale \& Lewis, 2004; Desrochers, Morissette, \& Ricard, 1995; Lock, Young, Service, \& Chandler, 1990; Vygotsky, 1978).

One main diagnostic criterion for the presence of communicative intent as suggested by Bates, Camaioni, and Volterra (1975), is infants' gaze alternation with the recipient. In favour of the claim that there is a developmental progression from non-communicative pointing for self to communicative pointing, Bates and colleagues reported that infants initially often point without looking at the recipient, and thus interpreted this as evidence for an initial absence of communicative intent. Further support for this idea derived from a longitudinal observational study with assessments

\footnotetext{
* Corresponding author.

E-mail address: liszkowski@eva.mpg.de (U. Liszkowski).
} 
at 12 and 15 months which found that only 15- but not 12-month olds reliably alternated gaze with a recipient when pointing (Desrochers et al., 1995; but see Carpenter, Nagell, \& Tomasello, 1998, who found that half of 12-month olds alternated gaze with a recipient when pointing). However, Liszkowski (2006) has argued that gaze alternation may not be the best criterion for establishing infants' communicative intent when pointing, because it is possible that infants either (1) look at the other person without communicative intent simply to check what she is doing, or (2) have communicative intent but do not look, instead assuming a communicative frame or relying on auditory instead of visual information. Following this argument, infants' looks may thus not be a valid marker for communicative intent, and so previous studies in favour of communicative interpretations (e.g., Camaioni et al., 2004; Carpenter et al., 1998; Franco \& Butterworth, 1996) may in fact be more susceptible to non-communicative interpretations than previously thought. Two recent studies have shown that when an adult reacted communicatively to infants' pointing by sharing attention and interest, infants looked less at the adult than when the adult did something unexpected like misidentifying the referent or not sharing infants' interest (Liszkowski, Carpenter, Henning, Striano, \& Tomasello, 2004; Liszkowski, Carpenter, \& Tomasello, 2007).

Another way to establish infants' communicative intent is to investigate infants' dissatisfaction with a recipient's response (see Bates, 1979). Two experimental studies to date have revealed that 12 -month olds point differentially as a function of different adult responses, which thus does not support the view that infants point only for the self (Liszkowski et al., 2004, 2007). Instead, these studies suggest that infants have the communicative intent to affect a recipient for specific reasons and persist if their intent is not satisfied. However, in these studies it is also possible that infants' communicative intent was apparent only through the adult's co-construction or scaffolding of her reactions after infants' pointing, but not from the outset of the pointing act.

In the current study, we therefore employed an even more stringent criterion for investigating communicative intent in infants' gestural communication. We asked whether infants understand that their pointing is effective only if a recipient can see it, that is, whether infants consider others' visual availability before pointing. One suggestive piece of evidence that this understanding may develop only later in ontogeny is Franco and Butterworth's (1996) finding that infants' looking at a recipient becomes progressively attuned to their pointing in the first half of their second year, such that 12-month olds look to a recipient only after they have pointed, 14-month olds look during their pointing, and only 16-month olds look to the recipient before they point. Liszkowski et al. (2004), too, found that 12-month olds look less to the recipient before than during/after they point. Both of these results may therefore suggest that infants initially do not know about the conditions under which their communication in the visual modality can be effective. Still, as cautioned above, infants' looking may be influenced by a variety of factors, and so it is important to investigate infants' understanding using other measures. In the current study, instead of measuring infants' looking, we thus used the more direct measure of pointing and point repetitions in order to investigate whether infants understand that their pointing is effective only if the recipient can see it. Our main question was whether infants would point at all - and whether they would repair their failed communication by then repeating their point - differentially as a function of the recipient's visual availability.

Interestingly, when a visual signal cannot be effective, that is, when the recipient's visual attention is not already available, another question is whether infants would somehow attempt to get the recipient to look at their visual signal. Infants' pointing is frequently accompanied by vocalizations, usually one-syllabic, vocalic utterances like 'ah' or 'da'. When the recipient is not looking at them, infants could vocalize in order to orient the recipient's visual attention to their gesture. That is, infants may combine a vocal and a gestural signal such that the vocalization elicits attention to their pointing gesture, and the pointing gesture then directs attention to the referent. In contrast, another possibility is that infants vocalize about the referent itself in order to orient the recipient's attention to it directly. In this case, both vocal and gestural communication would be object-related, and the vocalizations would serve to emphasize infants' communicative intent. Still another possibility is that pre-linguistic infants' vocalizations are not yet under their intentional control at all and rather occur as a function of environmental stimulation, arousal, or effort. Another question of this study was thus whether 1-year-old infants use their vocalizations intentionally when pointing at interesting events, and if so, whether they vocalize to elicit attention to their gesture, or instead to direct attention to what they point at, emphasizing the referent more generally.

We elicited pointing from 12- and 18-month-old infants under different social conditions. In one condition, an adult attended to the infant and was available for visual communication, as in all other studies of declarative pointing. We then measured how often infants pointed to interesting events that took place behind the adult. If infants pointed, the adult then asked for clarification while continuing to look to the infant, but never to the referent, in order to see whether 
infants would persist in their communicative attempt (see Camaioni et al., 2004, for a somewhat similar procedure). In the other, crucial condition, when the event took place behind the adult she attended not to the infant but instead always sideways to a book, and was thus unavailable for visual communication. For comparison, we also administered baseline trials in which the adult was available for visual communication and, if infants pointed, then reacted typically, by sharing attention and interest.

We expected that if infants understand something about the conditions under which visual communication can be effective, they should point less in the condition in which the adult is not available for visual communication than in the condition in which she is available. A further sign of their communicative intent would be more repairs - as evidenced by repetition of pointing - when their communicative intent was not satisfied (in the experimental conditions, again especially when the adult was visually available) than when it was satisfied (in the baseline trials). With regard to vocalizations, if infants vocalize self-referentially to call adults' attention to their gesture, they should vocalize more when the adult is not available for visual communication, in order to draw her attention to their pointing, and they should vocalize even before initiating the point. Alternatively, if infants vocalize to augment their gestural communication and orient adults' attention directly to the referent, infants should vocalize more in both experimental conditions, in which the adult asked for clarification, than in the baseline trials in which the adult successfully shared attention and interest, in order to persist in their referential intent by augmenting the signal. However, if the vocalizations that accompany infants' points are not yet under intentional control, there should be no differences in infants' vocalizations between experimental conditions and baseline trials.

\section{Method}

\subsection{Participants}

Infants were recruited from a database of German infants whose parents had expressed interest in participating in studies of child development. Infants were healthy, full-term babies who came predominantly from middleclass backgrounds. When contacted, parents were asked whether their infant already pointed. Fifty 18-month-old infants and thirty-eight 12-month-old infants who were reported to point were tested. Some infants were excluded because of fussiness or distraction (fourteen 18-month olds, six 12-month olds) or experimenter errors (four 18-month olds). The final sample thus included 64 infants: thirty-two 18-month olds (mean age: 18;16; range: $18 ; 0$ to $19 ; 0 ; 18$ girls, 14 boys) and thirty-two 12-month olds (mean age: 12;15; range: 12;1 to 12;28; 17 girls, 15 boys).

\subsection{Set-up and materials}

Testing took place in a $2.5 \mathrm{~m} \times 4 \mathrm{~m}$ testing room. The infant was seated in an infant-sized chair in front of a large screen made of white cloth sheets, which blocked the entire back of the testing room. The infant faced this screen from a distance of $2 \mathrm{~m}$. The screen had eight openings (flaps or slots) through which stimuli were protruded one at a time. Four openings were on the left and four were on the right side of the infant. The openings (six of them $60 \mathrm{~cm}$ and two of them $100 \mathrm{~cm}$ from the floor) were symmetrically positioned at about $45^{\circ}, 30^{\circ}(2 \times)$ and $25^{\circ}$ left and right from the infant's midline. A total of 10 stimuli were used, one for each trial. Six different hand puppets, two different marionettes, a lamp, and an electronically activated dancing pig served as stimuli. The two electronic stimuli were positioned on the floor in front of the screen at approximately $30^{\circ}$ to the infant's left and right (see Fig. 1).

The main experimenter (E1) sat between the infant and the screen, with her back to the screen and facing the infant from a distance of about $60 \mathrm{~cm}$. There was a small table between the infant and E1. Attached to this table was a toy with large, moveable beads with which the infant and E1 played between test trials to establish and maintain a social interaction. E1 also had a picture book with detailed farmyard scenes that she shared with the infant before experimental trials. The infant's parent was present, but was seated about $2 \mathrm{~m}$ behind the infant ( $45^{\circ}$ to the infant's left). An assistant (E2) remained invisible to the infant behind the screen. Two cameras filmed the infant from behind the screen (one on each side of the infant) and two cameras filmed E1 and the stimuli from behind the infant. E2 could watch E1 and the infant on a monitor behind the screen. E2 signalled the onset of infants' points and the end of the test period to E1 with a soft clicking noise made by pressing a button on a remote control device. 


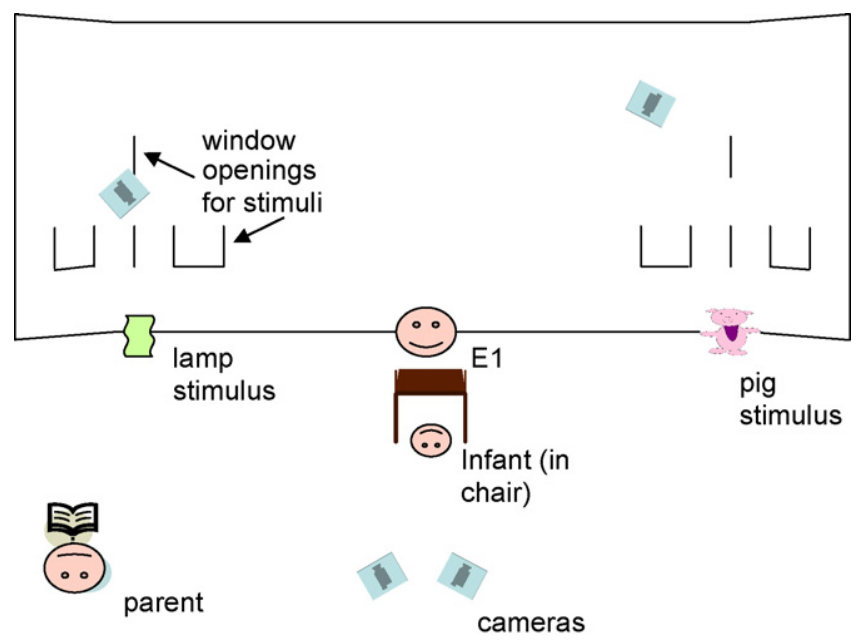

Fig. 1. Schematic drawing of the set-up.

\subsection{Procedure}

During extensive play between E1 and the infant in a warm-up area, parents were instructed by E2 not to initiate any communicative behavior toward the infant during testing and not to look at the screen at any time. The session began with a short play period with the bead toy on the table to keep the infant interested in E1 as a social partner. When E1 judged that the infant was relaxed and attentive, she gradually withdrew from the interaction and signalled to E2 by casually touching her ear to protrude or activate a stimulus. For all infants, the first trial was a baseline trial. While the stimulus was activated, E1 looked at the infant and ignored the stimulus until the infant pointed; then she reacted immediately and continuously for $10 \mathrm{~s}$ with joint attention, that is, she repeatedly looked back and forth between the stimulus and the infant's face, talking excitedly about the stimulus and commenting on the fact that they were seeing it together. For example, she said something like: "Oh, it's Tigger! Look at his stripes! And look how he's waving at you!" Then, after $10 \mathrm{~s}$, the stimulus was withdrawn and the trial was over. There were five of these baseline trials in each session. Baseline trials, which were identical for all participants, alternated with five experimental trials in one of two conditions (see below), for a total of 10 trials. The stimuli were presented in two orders counterbalanced across subjects, such that baseline and experimental trials across subjects involved the same stimuli. Between trials, E1 and the infant resumed the normal play interaction with the bead toy.

Before each experimental trial, in both experimental conditions E1 held up an open picture book to her side (the side opposite to that on which the stimulus was going to appear) looking back and forth from the infant to the book and commenting about the pictures in the book. E1 then signalled to E2 that the trial could begin and stopped talking about the book (but still kept it up in place at her side). Infants were randomly assigned to one of two experimental conditions. These conditions differed according to what E1 was doing when the stimulus was activated during the experimental trials. In the available condition, while E2 activated the stimulus for $10 \mathrm{~s}$, E1 looked at the infant the whole time and ignored the stimulus (and the book). In response to the infant's first point (see Liszkowski et al., 2004), she reacted immediately by asking "Hmm?...What?...What's there?...Hmm?" but still ignored the stimulus, looking straight at the infant. In the unavailable condition, the procedure was identical to that of the available condition except that E1 looked only at the picture book (and never at the infant's face). That is, while E2 activated the stimulus for $10 \mathrm{~s}$, E1 continued looking at the book she had just been sharing with the infant, ignoring the infant and the stimulus. If the infant pointed, E1 said the same words in the same tone while still turned toward the book, as if trying to figure out what a picture in it was. In both experimental conditions, E1 thus ignored the stimulus completely. Ten seconds after the infant's first point, the stimulus was removed or deactivated and E1 turned back to the infant, put the book down, and resumed a normal play interaction with the bead toy.

If an infant did not point at the stimulus during a trial, the stimulus was removed or deactivated after the infant had seen it but appeared uninterested and inattentive to it for more than $3 \mathrm{~s}$. In these cases, E2 then clicked the remote 
control to make it clear to E1 that the trial was over. She did this in all trials, even in the ones in which E1 could see the infant herself. Infants hardly seemed to notice this sound and were not distracted by it.

\subsection{Coding and reliability}

We were primarily interested in infants' points and vocalizations. Coding was done with a specialized computer program with on/off activation of a keyboard key corresponding to the occurrence and duration of the relevant behaviors. Points were coded when the infant extended the arm (either fully or slightly bent) and index finger or open hand, palm down, in the direction of the stimulus. Point duration was coded from the onset of the movement which resulted in a point until the arm was drawn back and the index finger or hand was no longer extended. Only points to the stimuli were coded. Vocalizing was coded independent of potential semantic content and only when it was communicative, that is, when it was directed at E1 or the stimulus and not vegetative or fussing. We used the following measures, calculated separately for baseline and experimental trials:

Trials with a point: The number of trials in which infants pointed at least once as a proportion of the total number of trials.

Point repetitions: The number of trials in which infants pointed more than once as a proportion of the trials in which infants pointed at least once.

Duration of points: Duration in seconds of pointing divided by the number of points.

Latency to point: The mean time interval in seconds between infants' first look to the stimulus and their first point.

Number of vocalizations before pointing: The mean number of communicative vocalizations after the first look to the stimulus and before the first point.

Number of vocalizations during and after pointing: The mean number of communicative vocalizations during and after the first point until the end of the trial.

Duration of vocalizations before pointing: The mean duration of communicative vocalizations after the first look to the stimulus and before the first point.

Duration of vocalizations during and after pointing: The mean duration of communicative vocalizations during and after the first point until the end of the trial.

Inter-observer reliability was assessed by a second coder who was naïve to the hypotheses of the study, who coded $25 \%$ of the infants' tapes across conditions. Agreement for presence/absence of pointing and point repetitions in each trial was excellent: Cohen's $\kappa$ 's were .93 and .91 , respectively. For the measures duration of pointing, latency to point, number of vocalizations, and duration of vocalizing, the coders' means for each infant were highly correlated (Spearman's $\rho$ 's, all $p$ 's <.001) and did not differ significantly from each other (paired $t$-tests): $r=.944, t(15)=.589$; $r=.938, t(15)=.810 ; r=.990, t(15)=.966 ;$ and $r=.865, t(15)=1.10$, respectively.

\section{Results}

Twenty trials across thirteen 18-month olds and 25 trials across eleven 12-month olds were excluded from all analyses because on those trials infants were fussy or distracted ( 25 trials), or one of the Es committed procedural errors (20 trials). We report two main analyses on each of our measures. First, we tested whether the experimental conditions were different from each other (between-subjects), to see whether E1's visual availability had an effect on infants' gestural and vocal communication. Second, we tested whether each type of experimental condition was different to its baseline trials (within-subjects), to see whether infants would differentially persist in their communicative intent depending on whether it was satisfied or not. The measure latency to point did not yield any significant effects.

\subsection{Trials with a point}

Fig. 2 shows the proportion of trials with a point in each experimental condition and in the baseline trials for each group of participants separately. First, an ANOVA on the experimental trials with age group and experimental conditions as between-subjects factors revealed main effects of condition and age: infants pointed on significantly more trials in the available than unavailable condition, $F(1,60)=7.97, p=.006, \eta^{2}=.117$, and 18-month olds pointed overall on significantly more trials than 12 -month olds, $F(1,60)=9.35, p=.003, \eta^{2}=.135$, with no interaction with age. Infants thus initiated less pointing when the adult was not looking at them than when she was. 


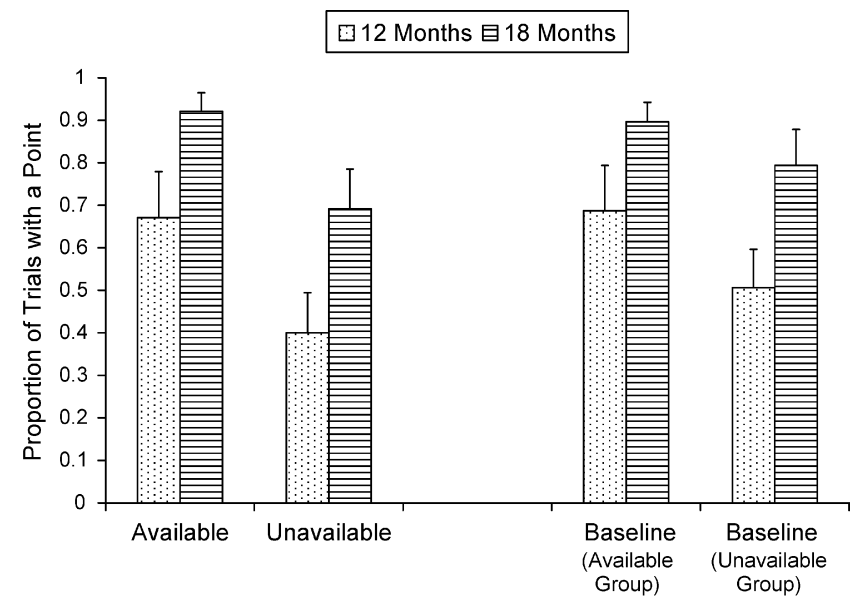

Fig. 2. Mean proportion of trials with a point in each experimental condition and their associated baseline trials.

Second, ANOVAs on each experimental condition separately, with experimental versus baseline trials as withinsubjects factor and age as between-subjects factor, revealed that in the unavailable condition infants pointed on significantly fewer experimental than baseline trials, $F(1,30)=7.22, p=.012, \eta^{2}=.194$, with no main effect of or interaction with age. For the available condition, in which E's visual orientation before pointing was similar to the baseline trials there were no differences in infants' initiation of points between experimental and baseline trials. Thus, again infants initiated less pointing when the adult was not looking at them (i.e. unavailable condition) then when she was (i.e. baseline trials).

\subsection{Point repetitions}

Fig. 3 shows infants' point repetitions within the experimental and baseline trials (again, for each experimental group separately). First, an ANOVA on the experimental trials with age group and experimental conditions as betweensubjects factors revealed that infants repeated their points in more trials in the available condition, when the adult could see them than in the unavailable condition, when she could not, $F(1,49)=5.99, p=.018, \eta^{2}=.109$, with no main effect of or interaction with age.

Second, ANOVAs on each experimental condition separately, with experimental versus baseline trials as withinsubjects factor and age as between-subjects factor, revealed that infants repeated pointing significantly more in both available and unavailable conditions compared to their respective baseline trials, with no main effect of or interaction with age: $F(1,25)=16.24, p<.001, \eta^{2}=.394 ; F(1,22)=4.87, p=.038, \eta^{2}=.181$, respectively. Thus, infants repeated their pointing more when their communicative intent was not satisfied than when it was.

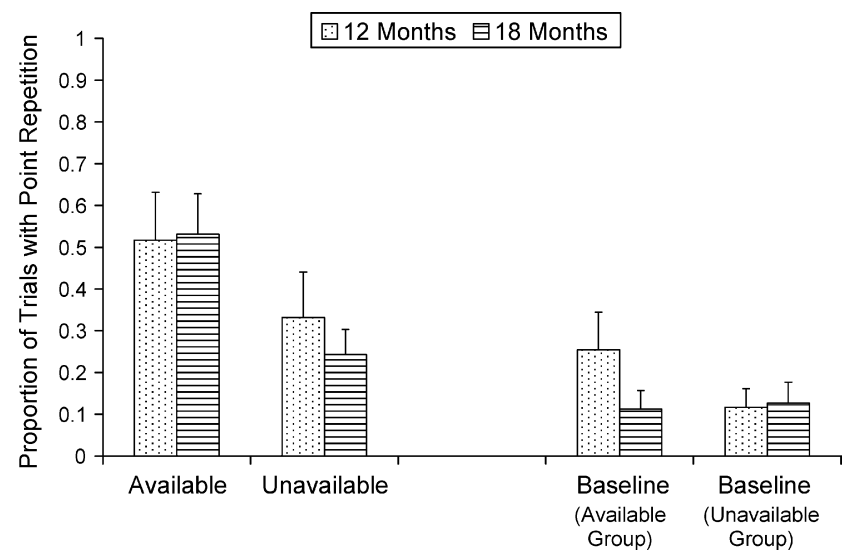

Fig. 3. Mean proportion of trials with point repetitions in each experimental condition and their associated baseline trials. 


\subsection{Duration of points}

First, an ANOVA on the experimental trials with age group and experimental conditions as between-subjects factors revealed an interaction of age and condition, $F(1,49)=4.67, p=.036, \eta^{2}=.088$, such that 18-month-olds' points were longer in the unavailable than the available condition (means $=5.27 \mathrm{~s}[$ S.D. $=4.40]$ vs. $3.59 \mathrm{~s}[$ [S.D. $=1.67]$ ) but $12-$ month-olds' points were shorter $($ means $=2.39 \mathrm{~s}[$ S.D. $=1.80]$ vs. $4.04 \mathrm{~s}[$ S.D. $=2.14]$ ). These differences were near the conventional significance level for the 12-month olds $(p=.061$, two-tailed) but not for the 18-month olds $(p=.166$, two-tailed).

Second, ANOVAs on each experimental condition separately, with experimental versus baseline trials as withinsubjects factor and age as between-subjects factor, revealed for the available condition no significant effects, and for the unavailable condition an interaction of trial type and age, $F(1,22)=6.06, p=.022, \eta^{2}=.216$, such that 18 -montholds' points were longer in the unavailable condition than in its baseline trials (means $=5.48$ [S.D. $=4.50]$ vs. 3.44 [S.D. = 3.22]) but 12-month-olds' points were shorter $($ means $=2.39$ [S.D. $=1.80]$ vs. 3.93 [S.D. = 2.78]). For each age group separately, the differences were significant for the 12-month olds $(t(10)=2.93, p=.015$, two-tailed) but not for the 18-month olds ( $p=.131$, two-tailed). Taken together, both interactions thus revealed a developmental difference such that 12-month-olds' pointing was shorter relative to 18-month olds' when E did not look at them than when she did.

\subsection{Vocalizing}

First, ANOVAs on the experimental trials with age group and experimental conditions as between-subjects factors yielded no significant differences between conditions or age groups for the mean number or duration of vocalizations before infants' points. Thus, when E was not looking at them infants did not use their vocalizations systematically to elicit E's attention before pointing. The same analyses on the vocalizations during/after infants' points also yielded no significant differences between conditions, but revealed significant age differences for both number and duration of vocalizations, such that 18 -month olds vocalized overall more than 12 -month olds: $F(1,49)=5.04, p=.029, \eta^{2}=.100$ for number and $F(1,49)=9.57, p=.003, \eta^{2}=.163$ for duration, see Figs. 4 and 5, respectively.

Second, ANOVAs on each experimental condition separately with experimental versus baseline trials as withinsubjects factor and age as between-subjects factor yielded no significant differences for either condition or either age group for the mean number or duration of vocalizations before infants' points. Thus, again infants vocalized as much when E was looking away as when she was looking at them (in the baseline trials before the point). However, the same analyses on the number of vocalizations during/after infants' first point revealed that infants vocalized more often in both experimental conditions compared to their baseline trials (available condition: $F(1,25)=24.68, p<.001, \eta^{2}=.497$; unavailable condition: $F(1,22)=12.29, p=.002, \eta^{2}=.358$ ). These effects both interacted with age (available condition:

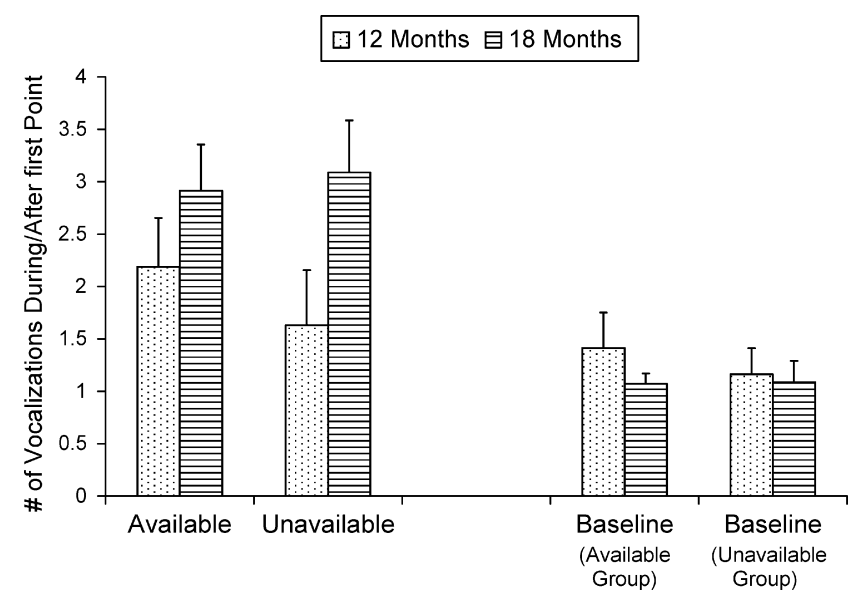

Fig. 4. Mean number of vocalizations during/after infants' first point in each experimental condition and their associated baseline trials. 


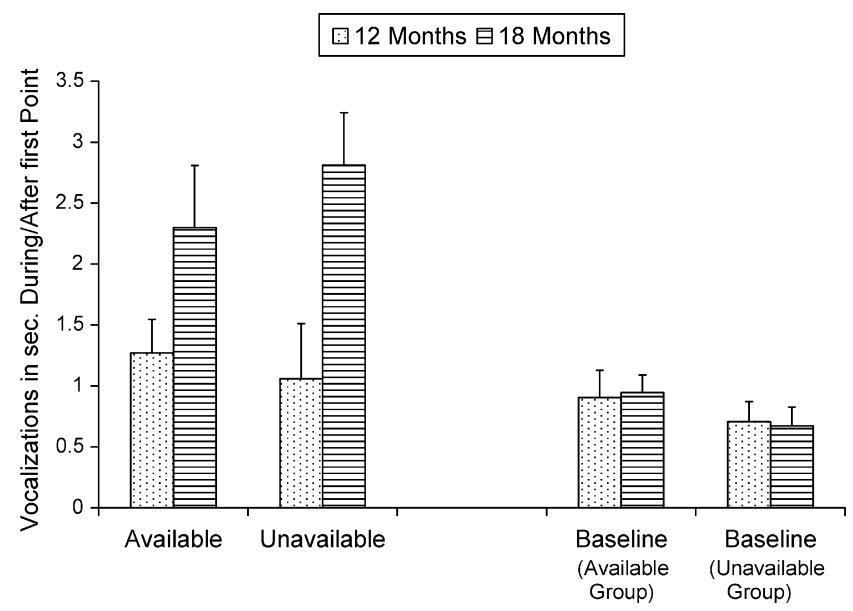

Fig. 5. Mean duration of vocalizations during/after infants' first point in each experimental condition and their associated baseline trials.

$F(1,25)=3.70, p=.066, \eta^{2}=.129$; unavailable condition: $\left.F(1,25)=6.26, p=.020, \eta^{2}=.222\right)$. Simple effects (Fisher's PLSD, based on the variance of the overall ANOVA) revealed that 18-month olds vocalized significantly more often in both experimental conditions compared to their baselines (mean differences 1.84 for availability condition, and 2.10 for unavailability condition, both $p$ ' $s<001$, two-tailed). Twelve-month olds also vocalized more often in the available condition compared to the baseline trials, but not in the unavailable condition (mean differences: $.81, p=.059$, and .35 , $p>.500$, respectively, $p$ 's two-tailed).

For duration of vocalizing during/after infants' first point, infants vocalized on average longer in both experimental conditions compared to their baseline trials (available condition: $F(1,25)=9.09, p=.006, \eta^{2}=.267$; unavailable condition: $\left.F(1,22)=21.51, p<.001, \eta^{2}=.494\right)$. Only in the unavailable condition did this interact with age $(F(1$, $22)=13.26, p=.001, \eta^{2}=.376$ ). Simple effects (Fisher's PLSD, based on the variance of the overall ANOVA) revealed that 18 - but not 12 -month olds vocalized significantly longer in the unavailable condition compared to the baseline (mean difference $2.25 ; p<.001$, two-tailed). This pattern was the same for the available condition (mean difference $1.35 ; p<.001$, two-tailed). Taken together, 18 -month olds vocalized more often and longer during/after their first point when their communication was unsuccessful (i.e. in the experimental conditions) than when it was successful (i.e. in the baseline trials). For the 12-month olds, a similar pattern was seen especially for number of vocalizations in the availability condition.

\subsection{Vocalizing and point repetitions}

In order to investigate in detail what infants were doing with their vocalizing when they repeated their pointing in the experimental conditions, we conducted ANOVAs on the number and duration of vocalizations during/after infants' first point in those trials in which infants repeated pointing. This yielded significant main effects of age such that 18-month olds vocalized both more $\left(F(1,34)=4.88, p=.034, \eta^{2}=.126\right)$ and longer $\left(F(1,34)=15.09, p<.001, \eta^{2}=.307\right)$ in these trials than 12-month olds. For the measure duration there was a small but non-significant interaction of condition and age $(p=.109)$ suggesting that only the 18 -month olds vocalized longer in the unavailable than available condition $(F(1$, 19) $=4.25, p=.053, \eta^{2}=.183$; see Fig. 6).

Second, ANOVAs on each experimental condition separately with experimental versus baseline trials as withinsubjects factor and age as between-subjects factor revealed that in the available condition infants vocalized significantly more $\left(F(1,8)=14.03, p=.006, \eta^{2}=.637\right)$ and longer $\left(F(1,8)=6.38, p=.035, \eta^{2}=.444\right)$ when they repeated pointing compared to the baseline. In the unavailable condition, this pattern was significant for the number of vocalizations $(F(1$, $\left.7)=7.64, p=.028, \eta^{2}=.522\right)$, but not for the duration $\left(F(1,7)=3.67, p=.097, \eta^{2}=.344\right)$. Thus, again infants increased their vocalizations when their communicative intent was not satisfied (i.e. in the experimental conditions as compared with the baseline trials). Too few infants repeated pointing in the baseline trials to make statistical comparisons between the age groups possible. 


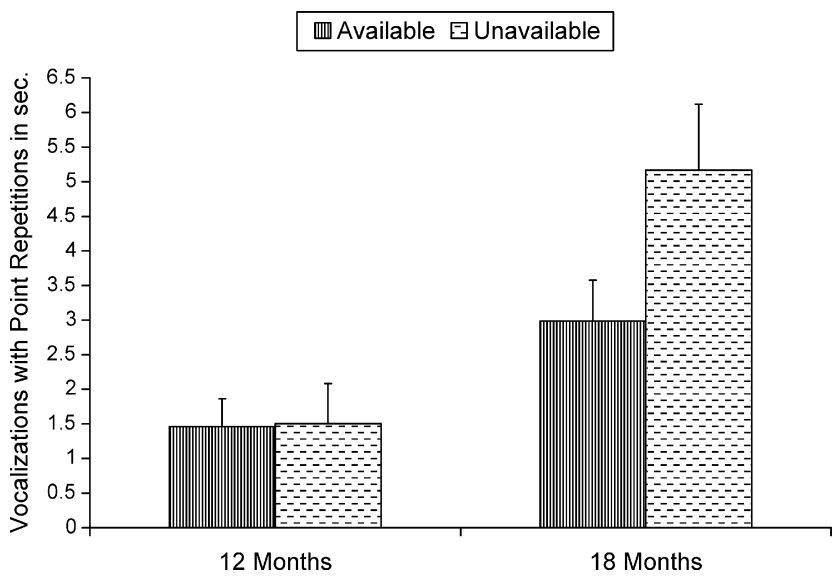

Fig. 6. Mean duration of vocalizations accompanying point repetitions in each experimental condition.

\subsection{Between-group comparison of baseline trials}

Finally, to make sure that any differences between conditions were not due to group differences generally we compared the baseline trials of both experimental groups with each other. The baseline trials followed the exact same procedure in both experimental groups so there should have been no differences between them. Indeed, we found no significant differences between the two groups' baseline trials on any measure, thus confirming that there were no general group differences.

\section{Discussion}

The current study investigated infants' communicative use of pointing and vocalizations accompanying their points. Results show that already at 12 months infants tailor their gestural communication in the visual modality to a partner's visual availability. Further, results show that infants intentionally use the vocalizations accompanying their pointing to emphasize their communicative intent when it is not satisfied. For the older infants there was some limited support for the view that infants may use their vocalizations to elicit attention to their visual gestures.

More specifically, with regard to pointing, the main result was that infants at both ages were more likely to point when an adult's visual attention was available for them (both in the available condition and the baseline trials) than when it was not (in the unavailable condition). In addition, infants repeated their pointing more often when their communicative intent was not satisfied (in the experimental conditions-again, more often when the adult's attention was available) than when the adult responded to them as expected (in the baseline trials). Importantly, the differences between conditions cannot be explained by a general lack of social interaction or infants' disengagement with the experimenter in the unavailable condition, because our control analysis clearly shows that infants of both experimental groups interacted with E equally much in the baseline trials.

These results thus support the view that 12-month olds already point with communicative intent, even when using the more stringent criterion of differential pointing depending on the recipient's concurrent visual orientation instead of gaze alternation (e.g., Carpenter et al., 1998) or signs of dissatisfaction with the recipient's response after the point (Liszkowski et al., 2004, 2007). This also suggests that 12-month-olds' lack of gaze alternation (Desrochers et al., 1995) and limited looking to the recipient before pointing (Franco \& Butterworth, 1996; Liszkowski et al., 2004) need not necessarily be interpreted as an absence of communicative intent. Given the findings of the current study, which demonstrate that 12-month olds clearly point with communicative intent, it is possible that other studies relying only on gaze alternation have underestimated infants' communicative abilities. For example, it may simply be that younger infants look less often to the recipient because their attention is mostly captured by the referent, especially if it is an interesting event like a moving puppet suddenly appearing. Presumably infants' point-accompanying looks are influenced by many factors, not just infants' communicative intent. 
With regard to vocalizing, results were that infants vocalized more during/after their first points when the adult did not attend to the event to which they pointed (in the experimental trials) than when she shared attention and interest (in the baseline trials). This pattern was the same in trials with repeated pointing. Thus, infants used their vocalizations intentionally, augmenting or repairing their gestural communication with increased vocalizing if it was not successful (i.e. when the adult asked for clarification or did not look at the referent). For the 12-month olds, however, this was reliable only for one measure, number of vocalizations, and only in the comparison with the available condition in which the adult was looking at them. Perhaps younger infants augment or repair their communication predominantly when the recipient establishes eye contact (i.e. in the available condition), even when communicating auditorily, and are 'turned off' if the recipient does not look at them (see Csibra, 2003; Gómez, 2004).

There was little evidence that infants use their intentional vocalizations to elicit attention to their visual gestures. Most notably, infants at both ages did not vocalize more before pointing when the adult was unavailable for visual communication than when she was available; nor did they do this during/after their pointing. This would suggest that 1-year olds do not vocalize self-referentially to draw adults' attention to their gesture.

However, it is possible that infants vocalized in both experimental conditions equally as much, but for different reasons. That is, in the unavailable condition they may have vocalized more than in the baseline trials to elicit attention to the gesture and in the available condition they may have vocalized more than in the baseline trials to emphasize their attempt to direct attention to the referent. This possibility could thus explain the lack of differences between conditions if both conditions are different from the baseline trials. For the 12-month olds, however, results did not support this view, because they vocalized more compared to the baseline trials only in the available, not the unavailable condition. Thus, 12-month olds do not seem to use their vocalizing to elicit attention to their gestures. It is possible that 18-month olds used their vocalizations more flexibly. First, unlike 12-month olds, they did vocalize more when the adult was unavailable than in the baseline trials. Second, in trials with point repetitions their vocalizations tended to be longer when the adult did not look at them (in the unavailable condition) than when she did (in the available condition). Along these lines it may be interesting to note the interaction of point duration with age: In contrast to the 12-month olds, who showed the opposite pattern, 18-month olds pointed longer in the unavailable than available condition. Perhaps the 18-month olds tended to do this so that if the adult's attention was attracted by their vocalizing, she could then still see the pointing, while 12-month olds did not attempt to attract the adult's attention by vocalizing and so stopped their ineffective pointing more quickly.

Taken together, the results on point-accompanying vocalizations show that infants vocalize intentionally when pointing and use their vocalizations to repair communication. However, they use their vocalizations mainly to emphasize their overall communicative intent and draw attention to the referent rather than specifically to elicit visual attention to their gestural communication. Possibly around 18 months, perhaps due in part to the transition from pre-linguistic to linguistic communication, infants begin using their vocalizations more flexibly, also attempting to elicit attention to their gestures.

This new evidence for infants' communicative use of pointing and point-accompanying vocalizations calls into question whether the absence or specific timing of looks to a recipient is a valid criterion for denying communicative intent. Instead, a private, non-communicative function of pointing (see Delgado, Gómez, \& Sarriá, in press) should be established by positive evidence rather than absence of looks to a recipient. Then, the relation of communicative and non-communicative pointing in ontogeny could be investigated more thoroughly. For example, one possibility is that non-communicative pointing is not simply a precursor to communicative pointing (as Bates et al., 1975, suggested), but instead follows from it.

Another interesting question remaining for future research is when infants begin combining vocal and gestural communication in complementary ways such that one signal is about the other. Research has shown that during the transition from the pre-linguistic to the linguistic period in the second half of their second year, infants begin combining words and gestures in non-redundant ways (e.g., point to cup+ "drink" instead of point to cup+ "cup"), and that these non-redundant combinations predict the onset of two-word utterances (Capirci, Iverson, Pizzuto, \& Volterra, 1996; Iverson \& Goldin-Meadow, 2005). Further research following up on the current study of point-accompanying preverbal vocalizations may provide a window into the process of infants' differentiation of cross-modal communication and the emergence of sequential signal combinations over the second year of life. 


\section{Acknowledgements}

Thanks to Angela Loose, Antje Endesfelder, Nadja Richter, Kirsten Vinzelberg, Christiane Schwier and Dorothe Coenen for helping with the data collection and coding. This study was in part conducted as the second author's Diplom thesis at the University of Leipzig, Germany.

\section{References}

Bates, E. (1979). The emergence of symbols: Cognition and communication in infancy. New York: Academic Press.

Bates, E., Camaioni, L., \& Volterra, V. (1975). The acquisition of performatives prior to speech. Merrill Palmer Quarterly, 21, $205-224$.

Camaioni, L., Perucchini, P., Bellagamba, F., \& Colonnesi, C. (2004). The role of declarative pointing in developing a theory of mind. Infancy, 5(3).

Capirci, O., Iverson, J. M., Pizzuto, E., \& Volterra, V. (1996). Gesture and words during the transition to two-word speech. Journal of Child Language, $23,645-673$.

Carpendale, J., \& Lewis, C. (2004). Constructing an understanding of mind: The development of children's social understanding within social interaction. Behavioral and Brain Science, 27(1), 96-97.

Carpenter, M., Nagell, K., \& Tomasello, M. (1998). Social cognition, joint attention, and communicative competence from 9 to 15 months of age. Monographs of the Society for Research in Child Development, 63(4) [serial no. 255].

Csibra, G. (2003). Teleological and referential understanding of action in infancy. Philosophical Transactions of the Royal Society of London Series B: Biological Sciences, 358(1431), 447-458.

Delgado, B., Gómez, J. C., \& Sarriá, E. (in press). Private pointing and private speech: Developing parallelisms. In A. Winsler, C. Fernyhough, \& I. Montero (Eds.), Private speech, executive functioning and the development of self-regulation. Cambridge University Press.

Desrochers, S., Morissette, P., \& Ricard, M. (1995). Two perspectives on pointing in infancy. In C. Moore \& P. Dunham (Eds.), Joint attention: Its origins and role in development (pp. 85-101). Hillsdale, NJ: Erlbaum.

Franco, F., \& Butterworth, G. (1996). Pointing and social awareness: Declaring and requesting in the second year. Journal of Child Language, 23(2), $307-336$.

Gómez, J. C. (2004). Apes, monkeys, children, and the growth of mind. Cambridge: Harvard University Press.

Gómez, J. C., Sarria, E., \& Tamarit, J. (1994). The comparative study of early communication and theories of mind: Ontogeny, phylogeny, and pathology. In S. Baron-Cohen, H. Tager-Flusberg, \& D. J. Cohen (Eds.), Understanding other minds: Perspectives from autism (pp. 397-426). Oxford: Oxford University Press.

Iverson, J. M., \& Goldin-Meadow, S. (2005). Gesture paves the way for language development. Psychological Science, 16, 368-371.

Liszkowski, U. (2006). Infant pointing at twelve months: Communicative goals, motives, and social-cognitive abilities. In N. Enfield \& S. Levinson (Eds.), The roots of human sociality: Culture, cognition, and interaction (pp. 153-178). Oxford: Berg.

Liszkowski, U., Carpenter, M., Henning, A., Striano, T., \& Tomasello, M. (2004). Twelve-month-olds point to share attention and interest. Developmental Science, 7(3), 297-307.

Liszkowski, U., Carpenter, M., \& Tomasello, M. (2007). Reference and attitude in infant pointing. Journal of Child Language, 34, 1-20.

Lock, A., Young, A., Service, V., \& Chandler, P. (1990). Some observations on the origins of the pointing gesture. In V. Volterra \& C. J. Erting (Eds.), From gesture to language in hearing and deaf children (pp. 42-55). Berlin: Springer-Verlag.

Moore, C., \& Corkum, V. (1994). Social understanding at the end of the first year of life. Developmental Review, 14(4), $349-372$.

Tomasello, M., Carpenter, M., \& Liszkowski, U. (2007). A new look at infant pointing. Child Development, 78, 705-722.

Vygotsky, L. (1978). Mind in society: The development of higher psychological processes. Cambridge, MA: Harvard University Press. 